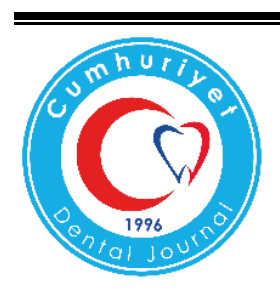

\title{
THE REGENERATIVE DENTISTRY: CURRENT APPROACHES AND FUTURE INSIGHTS
}

\author{
(D)Marco Tatullo ${ }^{1,2,3,4}$
}

ORCID ID: 0000-0001-7340-0708

1 Department of Basic Medical Sciences, Neurosciences and Sense Organs, University of Bari “Aldo Moro”, Italy

2 Marrelli Health - Tecnologica Research Institute, Biomedical Section, Street E. Fermi, Crotone, Italy

3 Department of Therapeutic Dentistry, Sechenov University Russia, Moscow, Russia

4 Honorary Senior Lecturer, Dundee University, Dundee, UK

Regenerative medicine typically aims to promote and improve the healing of tissues and organs of the human body, to restore the physiological architecture and the main functions lost. ${ }^{1}$

The combination of several scientific fields, including tissue engineering and cell biology, has led to the development of novel regenerative therapies that claim to obtain a better repairing, regenerating and replacing of tissues and organs damaged by chronic and acute diseases, or by severe traumatic injuries. In this landscape, the mesenchymal stem cells (MSCs) have been widely reported to self-regenerate and differentiate towards different tissues, under specific conditions and stimuli. ${ }^{2}$ Nowadays, MSCs are routinely used in autologous transplantation: the use of specific molecular effectors and biomimetic scaffolds populated with such MSCs has shown promising results both in vitro and in vivo experiments aimed to achieve a time-effective tissue reconstruction and a functional regeneration of complex organs.

Besides, studies on the extracellular matrix (ECM) and its interaction with cells have improved the knowledge of stem cell behavior and tissue growth and differentiation on different surfaces. Recent studies on novel 3D-cultured cell models have investigated specific pathways in "living functional conditions", combining ECMbased scaffolds, growth factors (GFs) and stem cells (SCs). Nevertheless, the last debates in the scientific community are discussing the usefulness of the traditional concept of scaffolds in modern regenerative procedures. ${ }^{3}$

This critical review aims to discuss the more inspiring and promising approaches with the novel biomimetic scaffolds, and about the last techniques used to regenerate tissues without the need to use the scaffolds.

The current regenerative procedures can recognize two main approaches: scaffold-based and scaffold-free.

The promising results achieved by regenerative procedures have significantly influenced various medical fields, including dentistry, and specifically the endodontics.

Regenerative dentistry aims to translate the latest discoveries in stem cell research, to regenerate and repair both soft and hard oral tissues. ${ }^{3}$

In the early ' 90 s, the regenerative techniques have started to use autologous matrices, like the

How to Cite: Tatullo M. Guest Editorial: The Regenerative Dentistry: Current Approaches and Future Insights. Cumhuriyet Dent J 2020:23:1:1-3. 
platelet concentrates and growth factors, to improve tissue regeneration and to speed the healing process. In the last decades, the development of new biomaterials has allowed scientists to combine the oral-derived mesenchymal stem cells (MSCs) and novel biomimetic scaffolds in dental repairing, so to induce not only a reparative process but also an immunomodulatory activity, improving the reparative process and the support of scaffolds to the dental implants. ${ }^{4}$

The regenerative procedures have been simplified thanks to the discovery of several MSC sources easily obtainable from the oral tissues; more in detail, the human teeth seem to have several structures rich in stem cells, characterized by easy harvesting and high efficiency during the isolation and culture stages, compared to other MSCs found in other tissues of human body. ${ }^{5}$

Undoubtedly, the most investigated oralderived MSCs are the dental pulp stem cells (DPSCs). ${ }^{6}$

Several studies have well demonstrated the important contribution of such stem cells towards the regenerative procedures and immunomodulation in dentistry. ${ }^{7-10}$

In 2003, MSCs from human exfoliated deciduous teeth (SHED) were suggested as a regenerative tool able to treat different gingival damages and to regenerate both dental and bone tissues. ${ }^{11}$ Furthermore, the human periodontal ligament stem cells (PDLSC) have been shown to have the ability to differentiate into cells similar to cementoblasts, to regenerate structures similar to dental cement that bind the periodontal ligament, thus contributing to the repairing of the whole periodontal organ. ${ }^{12}$

The discovery of MSCs in dental structures has created also interest in the anatomical tissues surrounding teeth: the fibrous/connective envelope around the developing tooth, the dental follicle, hosts MSCs named progenitor cells of the dental follicle (DFPC). ${ }^{13}$ Similarly, the apical gingival papilla surrounding the human teeth has a rich population of stem cells (SCAP). ${ }^{14}$ DFPC and SCAP can both differentiate towards odontogenic and osteogenic lineages, as well as adipose and neuron-like cells. ${ }^{15}$

In this landscape, Marrelli, Tatullo, and Paduano worked on a new concept of oral-derived stem cells, by investigating the biological properties of the discarded tissues harvested during the surgical removal of decayed and inflamed teeth. This novel approach was based on the reusing of biological wastes in regenerative medicine. In fact, the authors found that the inner layer of human periapical cysts (hPCy-MSC) were rich of MSCs, able to differentiate towards the classic osteo-/adipo-/condro-genic lineages; such hPCy-MSCs were also compared with DPSCs, showing a stronger commitment towards the bone reconstruction, similarly to the bone marrow stem cells BMSCs. ${ }^{16-18}$

Stem cells have shown significant potential in regenerative dentistry, not only for their proliferative and differentiating ability but also for the powerful events induced by specific factors secreted during their lifetime. Stem cells are, moreover, strategic players able to modulate the inflammatory response when they are engrafted in tissues, in combination with scaffolds or not. In the last few years, scientists are focusing their attention on the interaction between stem cells and the biological site of implantation: this interaction is mediated by several co-factors that work to reproduce a more "friendly" microenvironment, also called "stem cell niche". The establishment of these conditions is now considered fundamental to ensure successful engraftment of the newly implanted tissue/organ. The improvement of such conditions has developed a new concept of scaffold-free approach, which is different compared with the scaffold-based procedures. The use of human periapical cysts (hPCy-MSC) has been largely described: the impact of such novel and easy-to-obtain oral-/dental-derived MSCs has triggered the research on novel biomaterials and on innovative strategies for optimizing and translate in vivo the researches related to tissue engineering in dental applications. 


\section{REFERENCES}

1. Mao AS, Mooney,DJ. Regenerative medicine: Current therapies and future directions. Proc Natl Acad Sci USA 2015;112:14452-14459.

2. Tatullo M, Codispoti B, Pacifici A, Palmieri F, Marrelli M, Pacifici L, Paduano F. Potential use of human periapical cyst-mesenchymal stem cells (hpcymscs) as a novel stem cell source for regenerative medicine applications. Front Cell Dev Biol 2017;5:103.

3. Amrollahi P, Shah B, Seifi, A, Tayebi L. Recent advancements in regenerative dentistry: A review. Mater. Sci Eng C Mater Biol Appl 2016;69:1383-1390.

4. Tatullo M, Marrelli M, Scacco S, Lorusso M, Doria S, Sabatini R, Auteri P, Cagiano R, Inchingolo F. Relationship between oxidative stress and "burning mouth syndrome" in female patients: a scientific hypothesis. Eur Rev Med Pharmacol Sci 2012;16:1218-1221.

5. Tatullo M, Codispoti B, Pacifici A, Palmieri F, Marrelli M, Pacifici L, Paduano, F. Potential use of human periapical cyst-mesenchymal stem cells (hpcymscs) as a novel stem cell source for regenerative medicine applications. Front Cell Dev Biol 2017;5:103.

6. Gronthos S, Mankani M, Brahim J, Robey PG, Shi S. Postnatal human dental pulp stem cells (DPSCs) in vitro and in vivo. Proc Natl Acad Sci USA 2000;95:13625-13630.

7. Reynolds AJ, Jahoda CA. Cultured human and rat tooth papilla cells induce hair follicle regeneration and fiber growth. Differentiation 2004;2:566-575.

8. Barry M, Pearce H, Cross L, Tatullo M, Gaharwar AK. Advances in Nanotechnology for the Treatment of Osteoporosis. Curr Osteoporos Rep 2016;14:87-94.

9. Tatullo M. About stem cell research in dentistry: many doubts and too many pitfalls still affect the regenerative dentistry. Int J Med Sci 2018;15:16161618.

10. Kerativitayanan P, Tatullo M, Khariton M, Joshi P, Perniconi B, Gaharwar AK. Nanoengineered
Osteoinductive and Elastomeric Scaffolds for Bone Tissue Engineering. ACS Biomaterials Science \& Engineering 2017;27:95-104.

11. Miura M, Gronthos S, Zhao M, Lu B, Fisher LW, Robey PG, Shi S. SHED: stem cells from human exfoliated deciduous teeth. Proc Natl Acad Sci USA 2003;100:5807-5812.

12. Seo BM, Miura M, Gronthos S, Bartold PM, Batouli S, Brahim J, Young M, Robey PG, Wang CY, Shi S. Investigation of multipotent postnatal stem cells from human periodontal ligament. Lancet 2004;364:149-155.

13. Morsczeck C, Götz W, Schierholz J, Zeilhofer F, Kühn U, Möhl C, Sippel C, Hoffmann KH. Isolation of precursor cells (PCs) from human dental follicle of wisdom teeth. Matrix boil 2005;24:155-165.

14. Sonoyama W, Liu Y, Fang D, Yamaza T, Seo BM, Zhang C, Liu H, Gronthos S, Wang CY, Wang S, Shi $\mathrm{S}$. Mesenchymal stem cell-mediated functional tooth regeneration in swine. PloS One 2006;1:e79.

15. Yang B, Qiu Y, Zhou N, Ouyang H, Ding J, Cheng B, Sun J. Application of Stem Cells in Oral Disease Therapy: Progresses and Perspectives. Front Physiol 2017;8:197.

16. Marrelli M, Paduano F, Tatullo M. Cells isolated from human periapical cysts express mesenchymal stem cell-like properties. Int J Biol Sci 2013;9:10701078 .

17. Tatullo M, Falisi G, Amantea M, Rastelli C, Paduano F, Marrelli M. Dental Pulp Stem Cells and Human Periapical Cyst Mesenchymal Stem Cells in Bone Tissue Regeneration: Comparison of Basal and Osteogenic Differentiated Gene Expression of a Newly Discovered Mesenchymal Stem Cell Lineage. J Biol Regul Homeost Agents 2015;29:713-718.

18. Ballini A, Cantore S, Scacco S, Coletti D, Tatullo M. Mesenchymal Stem Cells as Promoters, Enhancers, and Playmakers of the Translational Regenerative Medicine 2018. Stem Cells Int 2018;2018:6927401. 